\title{
EDITORIAL
}

\section{Using Genomics to Improve Pigmented Lesion Management \& Health Outcomes}

\author{
Clay Cockerell, MD¹, Roger Ceilley $\mathrm{MD}^{2}$, Mark Lebwohl, $\mathrm{MD}^{3}$, Darrell Rigel, MD, $\mathrm{MS}^{3}$
}

1 University of Texas Southwestern Medical Center, Dallas, TX

2 University of lowa School of Medicine, Des Moines, IA

${ }^{3}$ Icahn School of Medicine at Mount Sinai, New York, NY

The advantage of a noninvasive test that reduces unnecessary biopsies is obvious. In managing pigmented lesions, US dermatologists biopsy about 5 million uncertain lesions annually to identify around 200,000 in situ and invasive melanomas (number needed to biopsy=25). ${ }^{1,2}$ Current practice relies on visual assessment with or without dermoscopy to determine which pigmented lesions warrant biopsy and histopathology.

The current issue of SKIN presents a costbenefit analysis of a non-invasive 2-gene expression profile (2-GEP) (Pigmented Lesion Assay [PLA] DermTech, La Jolla, $\mathrm{CA})$, which studies have demonstrated has a $>99 \%$ negative predictive value (NPV). ${ }^{3}$ With the ability to detect genomic atypia in pigmented lesions, this technology offers an additional tool to clinicians faced with the conundrum of determining whether a pigmented lesion should be biopsied/removed or if they can be safely monitored. ${ }^{4}$ The validity and utility of the test has been established in over 20 peer reviewed publications; however, its impact on healthcare delivery cost has to date only been considered in one earlier publication focused on Medicare patients. ${ }^{5}$ In this issue of SKIN, Siegel et al. provide an analysis of cost savings to commercial payors on this test that facilitates early detection and guides biopsy decisions. Their model demonstrates that adding detection of genomic atypia to visual assessment to make critical pre-biopsy management decisions has the potential to improve care while also reducing costs.

Siegel and colleagues present a Return on Investment (ROI) model from a US payor perspective to evaluate the potential savings to commercial health plans if genomic atypia detection with 2-GEP is incorporated into the current visual assessment/histopathology (VAH) pathway for pigmented lesions uncertain for melanoma. Using 2019 Optum Group claims data encompassing 239,854 patients with lesions suspicious for melanoma, costs were assessed through the pathway of initial visual assessment, surgical procedure(s), histopathology, and subsequent management. The ROI model predicted annual net savings of $\$ 0.54$ per member per month for commercial health plans over a three-year period with incorporation of the 2-GEP test into the VAH pathway. Their findings suggest that for a plan with 1 million covered lives, this translates into potential aggregate savings of $\$ 5.66$ million over three years. 
Siegel et al. also showed high rates of biopsies and excisions on benign lesions using the VAH pathway, which highlights the need for more accurate clinical assessment of pigmented lesions. In their analysis using this clinical setting, $95.7 \%$ of surgically assessed lesions clinically suspicious for melanoma were diagnosed as benign, and $30.4 \%$ of patients with benign lesions undergoing a more advanced procedure such as an excision, either initially or following a biopsy. Melanoma diagnosis rates were $0.9 \%$ with biopsy only, $0.1 \%$ with excision only, and $17.9 \%$ with a biopsy followed by excision procedures. With access to objective genomic information such as that provided by the 2-GEP test, there is the opportunity to better identify which patients are most likely to benefit from a procedure for further evaluation of their lesions.

These healthcare economic analyses are complemented by another recent publication in SKIN by Skelsey et al. on the performance of 2-GEP and its utility in intended use populations. ${ }^{6}$ That study confirmed a NPV $>99 \%$ in real world settings and a positive predictive value (PPV) of $18.7 \%$, which is an approximately 5 -fold improvement over visual assessment alone. ${ }^{7}$ Real-world NPV was determined by long-term follow-up of two cohorts of lesions that tested negative on the 2-GEP and were not initially biopsied. Out of 1,535 total lesions followed, 13 early-stage melanomas (7 in situ, 6 stage pT1a) were identified up to 3 years after the initial negative test result, corresponding to a real-world NPV of $>99 \%$. Real-world PPV was determined by identifying melanoma diagnoses among 316 lesions that tested positive on the 2-GEP within a US-based registry. Of the 3162 2GEP positive cases, 59 were diagnosed as melanoma by histopathology, corresponding to a PPV of $18.7 \%$. Of all 2-GEP positive lesions, $30.5 \%$ had histopathologic diagnoses corresponding to high-risk MPATH-Dx categories (Classes III-V, including severely dysplastic nevi, in situ melanomas, and invasive melanomas), demonstrating the ability of this technique to detect high-risk lesions such as dysplastic nevi with severe or high-grade atypia that are generally targeted for complete excision. Taken together, these data indicate that the 2-GEP test is highly reliable for ruling out melanoma and can also enhance our individual and collective number needed to biopsy to detect melanoma.

These 2 studies suggest that incorporation of 2-GEP analyses into the VAH pathway for assessing clinically suspicious pigmented lesions can result in savings for both CMS and commercial health insurance plans. ${ }^{5}$ Use of the 2-GEP improves patient care and improves health outcomes by using genomic assessments to minimize avoidable surgical procedures on benign lesions, enriching the population of melanomas within biopsied lesions, and decreasing downstream costs of late-stage melanoma diagnoses. For all of these reasons, creating broad access and coverage to transformative diagnostic technologies such as the 2-GEP is in the best interest of our colleagues, the healthcare system, and most importantly, our patients.

Conflict of Interest Disclosures: Dr. Rigel and Dr. Cockerell have served as consultants for DermTech.

Funding: None

Corresponding Author:

Darrell Rigel, MD, MS

Email: Darrell.rigel@gmail.com

\section{References:}

1. Anderson AM, Matsumoto M, Saul MI, Secrest AM, Ferris LK. Accuracy of Skin Cancer Diagnosis by Physician Assistants Compared With Dermatologists in a Large Health Care March 2022 Volume 6 Issue 2 
System. JAMA Dermatol. May 1

2018;154(5):569-573.

doi:10.1001/jamadermatol.2018.0212

2. Siegel RL, Miller KD, Fuchs HE, Jemal A. Cancer

Statistics, 2021. CA: A Cancer Journal for

Clinicians. 2021;71(1):7-33.

doi:https://doi.org/10.3322/caac.21654

3. Gerami P, Yao Z, Polsky D, et al. Development and validation of a noninvasive 2-gene molecular assay for cutaneous melanoma. J Am Acad

Dermatol. Jan 2017;76(1):114-120 e2.

doi:10.1016/j.jaad.2016.07.038

4. Data on file. DermTech, Inc. La Jolla, California.

5. Hornberger J, Siegel DM. Economic Analysis of a Noninvasive Molecular Pathologic Assay for

Pigmented Skin Lesions. JAMA Dermatol. Sep 1 2018;154(9):1025-1031.

doi:10.1001/jamadermatol.2018.1764

6. Skelsey M, Brouha B, Rock J, et al. Non-Invasive

Detection of Genomic Atypia Increases Real-

World NPV and PPV of the Melanoma Diagnostic

Pathway and Reduces Biopsy Burden. SKIN The

Journal of Cutaneous Medicine. 2021;5(5):512-

523.

7. Lott JP, Boudreau DM, Barnhill RL, et al.

Population-Based Analysis of Histologically

Confirmed Melanocytic Proliferations Using

Natural Language Processing. JAMA Dermatol.

Jan 1 2018;154(1):24-29.

doi:10.1001/jamadermatol.2017.4060 